\title{
Optimizing Power by Combining Small Cells with a Massive MIMO for Maximum Energy Efficiency under Two Path Loss Models
}

\author{
Teerapat Sanguankotchakorn \\ School of Engineering and Technology \\ Asian Institute of Technology \\ Pathumthani, Thailand \\ teerapat@ait.ac.th
}

\author{
Chintala Aravind Kumar \\ School of Engineering and Technology \\ Asian Institute of Technology \\ Pathumthani, Thailand \\ aravindch0406@gmail.com
}

\begin{abstract}
Currently, we are proceeding towards the $5^{\text {th }}$ generation of wireless networks. The 5G is expected to utilize very wide bandwidth and would have the greater capacity compared to $\mathbf{4 G}$. Therefore, the energy efficiency is one of the prominent roles in the design criterion. To achieve the maximum energy efficiency, the typical approaches are to increase the throughput or to decrease the power consumption. Recently, the approach to combine the small cell access points to Massive MIMO has been proved as one efficient way to achieve the maximum energy efficiency. In this work, the approach to combine two type of network, namely Small Cell Access Point (SCA) and Base Station (BS) with Massive MIMO, is considered for designing an energy efficient system. We investigate further this approach by extending the simulation to cover both 3GPP LTE as well as IMT 2020 environment with two different carrier frequencies under three scenarios. The performance is measured and compared with the existing work in terms of total power consumption per subcarrier of both the Base Station (BS) and Small Cell Access Points (SCAs). It is apparent that our proposed method provides the higher information rate at the same total power per sub carrier than the existing work. Additionally, general speaking, the 3GPP path loss model provides the lower total power consumption per sub carrier at BS than IMT-2020, while both path loss models almost has no impact on total power consumption at SCAs, regardless of carrier frequency and the number of antennas adopted in Massive MIMO at BS.
\end{abstract}

Index Terms-Massive MIMO, Optimization, Energy Efficiency

\section{INTRODUCTION}

The $5^{\text {th }}$ generation mobile networks $(5 \mathrm{G})$ is said to be the successor for present $4 \mathrm{G}$ communications. Since $5 \mathrm{G}$ would have a greater capacity comparing to $4 \mathrm{G}$ so that there would be greater density of population of mobile broadband users. $5 \mathrm{G}$ is expected to utilize very wide bandwidth upto several $\mathrm{GHz}$. The basic expectations of 5G are as follows [10]: (a) Data rates of $100 \mathrm{Mbps}$ for metropolitan areas for billions of users. (b) To have equal access of data for about $1 \mathrm{Gbps}$ for all people working on the same floor. (c) Parallel simultaneous connections in a huge number for wireless sensors. (d) Enhancement of spectral efficiency compared to $4 \mathrm{G}$ networks. (e) Increment of the coverage. (f) Reduction in latency compared to LTE (Long Term Evolution). According to the expectations of 5G networks, it can be anticipated that energy consumption is one important concern. It has been observed that the majority of greenhouse gas emission is from mobile ICT sector which is expected to be around 300 million tons of greenhouse gases per annum by the year 2020 [3]. So, to sustain the evolution in future $5 \mathrm{G}$ networks, it is very important to have a look on power consumption levels along with the service expectations.

In general, to obtain a system of maximal energy efficiency, the conventional approach would be to either increase the throughput or decrease the power consumption [8]. In this research, we follow the second approach that is to minimize the power consumption along with the preservation of Quality of Service (QoS). Here, we analyze the two-network combination approach, namely multiple antenna system motivated from Massive MIMO (MM) at the Base Station (BS) and Small Cell Access points (SCAs) by minimizing the total power consumption (static and dynamic) and satisfying QoS constraints [6], simultaneously. The approach of optimization proposed in [2] is adopted here. Furthermore, the system under our consideration is extended to cover two types of path loss models; 3GPP and IMT2020. The performance is evaluated by simulation under three scenarios in terms of total power consumption per subcarrier of both Base Station (BS) and Small Cell Access point (SCA) by varying the number of antennas at the Base Station. Our results are compared with the existing works [7].

The rest of the paper is structured as follows: Section II reviews the related works in the literature. Section III describes the proposed method as well as the analysis of the optimization algorithm. Section IV describes all parameters relevant to simulations. Section V illustrates the simulation results as well as the discussion while this work is concluded in Section VI.

\section{RELATED WORKS}

Massive MIMO (MM) is a multi-user MIMO technology consisting of a large number of base station (BS) antennas $M$ and providing service to $K$ single antenna user equipments (UEs) i.e $M \gg K$ [9]. Most of the works in the literature follows this model. For example, In [1], the merging massive 
MIMO with small cell is examined. Explanation about the architecture, performance measures, techniques and fusion of technologies, massive MIMO, small cell and summary about these technologies are reviewed. Energy efficiency and spectral efficiency problems are discussed.

Some works handle mainly the power consumption issue. In [2], the uplink energy efficiency maximization problem is formulated. The network densification plays a prominent role in achieving the high energy efficiency in cellular networks. The energy efficiency gain is achieved by suppressing the intra-cell interference and circuit power costs sharing among multiple users.

In [6], it is shown that the convex optimization problem can be solved by reducing spatial multi-flow transmission without compromising on QoS constraints. The optimal solution would be to assign users to either the BS or one of the SCAs as optimal transmitter. It is also illustrated that the power consumption can be improved by combining the two technologies of MM and SCAs. In [7], a low complexity algorithm of RZF beamforming technique for power allocation in massive MIMO SCA system is proposed. The convex optimization problem related to power allocation is solved. This study helps in getting motivated for spectral and energy efficient massive MIMO-SCA system which will be helpful for future wireless systems. In [9], the energy efficiency and spectral efficiency gains offered by MM over the current LTE networks are described. It also provides a brief information about how to boost the energy efficiency gains like scaling the number of base station antennas, low complexity operations, reducing $\mathrm{RF}$ chain requirements etc. The hybrid massive MIMO system is also explained.

\section{System Model AND ANALYSIS}

The system model consists of the combination of both massive MIMO (MM) and small cell access point (SCA) such that the power constraints at the SCA and base station (BS) are fulfilled without compromising on QoS constraints. This work analyzes the power optimization by employing $\mathrm{MM}$ at the base station (BS) and overlaying with small cell access points (SCAs). The lower complexity algorithm proposed by [7] is compared with MM-SCA system proposed in this work.

Actually, this combination of deployment is already done in [6]. It has 3 antennas at SCA in $3^{\text {rd }}$ generation partnership project (3GPP) standard's urban macro heterogenous deployment. However, in this work, the power consumption is optimized along with the goal of implementing the small cell and massive MIMO approach in different scenarios of International Mobile Telecommunications-2020 (IMT-2020) standard [4]. Two frequencies adopted in IMT-2020, namely $2 \mathrm{GHz}$ and $700 \mathrm{MHz}$ are considered.

\section{A. System Model}

A single cell downlink scenario where the macro base station has $N_{B S}$ number of antennas is considered. Those $N_{B S}$ antennas at BS deliver the information to $K$ single antenna users, where $N_{B S} \gg K$ is the basic working principle of

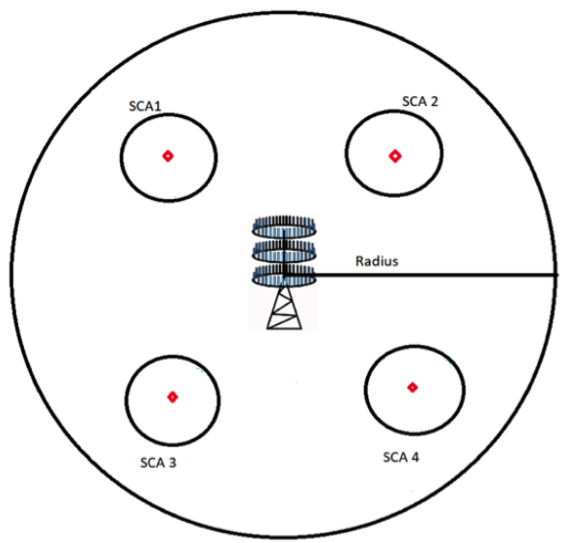

Fig. 1. System Model under consideration

massive MIMO. There are $S$ number of SCAs, where $S \geq 0$. Each of these SCAs has $N_{S C A}$ number of antennas where they form an overlay layer. One important thing to be observed here is that these SCAs have limited coverage area. This kind of arrangement will let the BS to cover the large area. The channels to $K$ users are modelled to be block fading, i.e., to have the fading process to be approximately constant for a number of symbol intervals. Assume that a single flat fading subcarrier in which the channels are represented in the baseband by $\mathbf{h}_{(k, 0)}^{H} \in C^{1 \times N_{B S}}$ and $\mathbf{h}_{(k, j)}^{H} \in C^{1 \times N_{S C A}}$ for the BS and the $j^{\text {th }}$ SCA, respectively. Now the received signal at user $k$ [6] can be written as

$$
y_{k}=\mathbf{h}_{(k, 0)}^{H} \mathbf{x}_{0}+\sum_{j=1}^{S} \mathbf{h}_{(k, j)}^{H} \mathbf{x}_{j}+n_{k}
$$

where transmitted signals are $\mathbf{x}_{0}$ and $\mathbf{x}_{j}$ at the BS and the $j^{\text {th }}$ SCA, respectively. The last term in (1) $n_{k} \sim C N\left(0, \sigma_{K}^{2}\right)$ refers to circularly symmetric gaussian noise measured in milliwatts $(m W)$.

Here the BS and SCAs are linked by a backhaul network which has a soft cell type allocation of resource, but for non-coherent linear communication which infers to be, information symbols would be emitted immediately but each user are served by multiple transmitters which is termed to be something like spatial multi-flow transmission. This would enable users covered by one SCA to receive additional signal from the other SCAs or BS.

\section{B. Problem Description}

Our main objective is to reduce the power consumption without being compromised on Quality of Service (QoS). QoS is defined as the information rate [bits/s/Hz] that must be achieved by each user parallelly, which can be defined as follows:

$$
\log _{2}(1+S I N R) \geq \gamma_{k}
$$

Here, $\gamma_{k}$ is the QoS target and $S I N R_{k}$ is given in (3) as follows [6]: 


$$
\operatorname{SIN} R_{k}=\frac{\left|\mathbf{h}_{(k, 0)}^{H} \mathbf{w}_{(k, 0)}\right|^{2}+\sum_{j=1}^{S}\left|\mathbf{h}_{(k, j)}^{H} \mathbf{w}_{(k, j)}\right|^{2}}{\sum_{(i=1, i \neq k)}^{K}\left(\left|\mathbf{h}_{(k, 0)}^{H} \mathbf{w}_{(i, 0)}\right|^{2}+\sum_{j=1}^{S}\left|\mathbf{h}_{(k, j)}^{H} \mathbf{w}_{(i, j)}\right|^{2}\right)+\sigma_{k}^{2}}
$$

where $\mathbf{w}_{(k, 0)}^{H} \in C^{N_{B S} \times 1}$ and $\mathbf{w}_{(k, j)}^{H} \in C^{N_{S C A} \times 1}$ represent the beam-forming vectors. Equation (3) provides the $k^{\text {th }}$ user's signal to interference plus noise ratio. The information rate $\log _{2}\left(1+S I N R_{k}\right)$ can be achieved by cancelling interference successively on symbols. Note that $\mathbf{w}_{(k, j)} \neq 0$ only for the $j^{\text {th }}$ transmitter that serves the $k^{\text {th }}$ user. The total power consumption is given by summation of dynamic power and static power, which are illustrated as follows [6]:

$$
\begin{gathered}
P_{\text {dynamic }}=\rho_{0} \sum_{k=1}^{K}\left\|\mathbf{w}_{(k, 0)}\right\|^{2}+\sum_{j=1}^{S} \rho_{j} \sum_{k=1}^{K}\left\|\mathbf{w}_{(k, j)}\right\|^{2} \\
P_{\text {static }}=\frac{\eta_{0}}{C} N_{B S}+\sum_{j=1}^{S} \frac{\eta_{j}}{C} N_{S C A}
\end{gathered}
$$

where the dynamic power consists of the emitted power $\sum_{k=1}^{K}\left\|\mathbf{w}_{(k, j)}\right\|^{2}$ multiplied by $\rho_{j} \geq 1$ which would represent the inefficiency of particular transmitter's power amplifier. Whereas the static power is directly proportional to $n_{j} \geq 0$ which is the power dissipation of filters, converters, mixers, baseband processing and number of antennas as well. It is normalized by $C \geq 1$ subcarriers. Every SCA and BS is prone to $L_{j}$ power constraints

$$
\sum_{k=1}^{K} \mathbf{w}_{(k, j)}^{H} \mathbf{Q}_{(j, l)} \mathbf{w}_{(k, j)} \leq q_{(j, l)} ; \quad l=1 \ldots L_{j}
$$

The positive semi-definite weighting matrices are $\mathbf{Q}_{(0, l)} \in C^{N_{B S} \times N_{B S}}$ and $\mathbf{Q}_{(j, l)} \in C^{N_{S C A} \times N_{S C A}}$ for $j=1, \ldots, S$ with order $N \times N \times L$ where $N$ and $L$ stands for total number of antennas and total number of power constraints, respectively. These parameters are defined per-array, per-antenna and constraints for soft shaping. We write $q_{(0, l)} \geq q_{(j, l)}$ for $1 \leq j \leq S$ because the coverage is provided by base station. Then, the optimization problem can be formulated as follows:

$$
\begin{array}{lc}
\begin{array}{ll}
\text { Minimize } \\
\mathbf{w}_{k, j} \forall k, j \\
\text { Subject to }
\end{array} & P_{\text {static }}+P_{\text {dynamic }} \\
& \log _{2}\left(1+S I N R_{k}\right) \geq \gamma_{k} \quad \forall k \\
& \sum_{k=1}^{K} \mathbf{w}_{(k, j)}^{H} \mathbf{Q}_{(j, l)} \mathbf{w}_{(k, j)} \leq q_{(j, l)} \forall j, l .
\end{array}
$$

Equation (7) is reformulated to explore as convex optimization problem in [6] since the complicated beamforming vectors makes the problem non-convex. The optimal solution is that there would be more than one transmitter to each user for MM-SCA system.

\section{Power Optimization Algorithm}

In order to reformulate into convex optimization problem, the semi-definite relaxation is used in [6] with the notation $\mathbf{W}_{(k, j)}=\mathbf{w}_{(k, j)} \mathbf{w}_{(k, j)}^{H} \forall k, j$.This notation has to be positive semi-definite with rank $\mathbf{W}_{(k, j)} \leq 1$. The problem formulated in (7) can be rewritten as follows:

$\begin{aligned} & \text { Minimize } \\ & \mathbf{W}_{(k, j)} \geq 0 \quad \forall k, j\end{aligned} \sum_{j=1}^{S} \rho_{j} \sum_{k=1}^{K} \operatorname{tr}\left(\mathbf{W}_{(k, j)}\right)+P_{\text {static }}$

Subject to

$$
\begin{gathered}
\operatorname{rank}\left(\mathbf{W}_{(k, j)}\right) \leq 1 \quad \forall k, j \\
\left.\sum_{j=1}^{S} \mathbf{h}_{(k, j)}^{H}\left[\left(1+\frac{1}{\gamma_{k}}\right) \mathbf{W}_{(k, j)}-\sum_{i=1}^{K} \mathbf{W}_{(i, j)}\right)\right] \mathbf{h}_{(k, j)} \geq \sigma_{k}^{2} \quad \forall k \\
\sum_{k=1}^{K} \operatorname{tr}\left(\mathbf{Q}_{(j, l)} \mathbf{W}_{(k, j)}\right) \leq q_{(j, l)} \forall j, l
\end{gathered}
$$

Equation (8) is a convex semi-definite optimization problem which can be solved by using a tool box called SEDUMI [5]. There are 3 possibilities for each $k^{t h}$ user which can be optimal solutions: (i) BS serving the $k^{\text {th }}$ user, (ii) The $j^{\text {th }}$ SCA serving the $k^{t h}$ user and (iii) Both BS and SCA serving the $k^{t h}$ user (very rare chances but there are some transition areas around each SCA where multi-flow transmission is utilized, since the SCA is unable to fully support the QoS targets). For which the proof is given in [6]. Usually, users who are close to SCA are served by SCA and those who are close to BS are served by BS.

The algorithm mentioned above gives us the modest complexity when $N_{B S}$ and $S$ grow large in number. For that not to happen, a low complexity power allocation algorithm has also been proposed [7], which would maximize the spectral efficiency and optimize the power consumption. An algorithm for how the power is being allocated is shown in [2], therefore, it is skipped here.

\section{Simulation Scenario AND Parameters}

\section{A. Simulation Scenario}

In this work, the simulation is carried out in order to measure the performance of MM-SCA system within three different environments as follows:

(i) Urban, Macro cell under 3GPP LTE standard

(ii) Urban, Macro cell, Non-Line-of-Sight deployment under IMT 2020 standard with $f_{c}=2 \mathrm{GHz}$

(iii) Urban, Macro cell, Non-Line-of-Sight deployment under IMT 2020 standard with $f_{c}=700 \mathrm{MHz}$

A single cell consisting of 10 active users randomly distributed in the macro cell is considered. Rayleigh small scale fading is assumed, $\mathbf{h}_{k, j} \sim C N\left(0, R_{k, j}\right)$.Correlation matrix is spatially uncorrelated among the $k^{t h}$ user and the $j^{\text {th }}$ SCA, where the reduced channel ranks and correlations of antenna are the main features. It must be noted that propagation loss for BS is different from SCA. 


\section{B. Simulation Parameters}

The simulation parameters used in this work are shown in Table I as follows:

TABLE I

SimUlation PARAMETERS [2]

\begin{tabular}{|l|l|}
\hline Definition & Value \\
\hline Carrier Frequency $\left(f_{c}\right)$ & $700 \mathrm{MHz}, 2 \mathrm{GHz}$ \\
\hline Number of Users & 10 \\
\hline Number of Antennas at BS & $20-100$ \\
\hline Number of Antennas at each SCA & $1-3$ \\
\hline Cell Radius & $0.5 \mathrm{~km}$ \\
\hline Small Cell Radius & $40 \mathrm{~m}$ \\
\hline Distance between BS and SCA & $0.35 \mathrm{~km}$ \\
\hline Number of Subcarriers & 300 \\
\hline Total Bandwidth & $10 \mathrm{MHz}$ \\
\hline Sub-carrier Bandwidth & $30 \mathrm{KHz}$ \\
\hline Noise Variance & $-127 \mathrm{dBm}$ \\
\hline Penetration Loss & $20 \mathrm{~dB}$ \\
\hline 3GPP Standard Urban Path Loss & $148.1+37.6 \log (d) d B$ \\
\hline $\begin{array}{l}\text { 3GPP Standard Deviation of Log- } \\
\text { normal Shadowing }\end{array}$ & $7 \mathrm{~dB}$ \\
\hline $\begin{array}{l}\text { IMT2020 Path Loss (Urban NLOS } \\
\left.\text { at } f_{c}=2 \mathrm{GH} z\right)\end{array}$ & $199.5+39.09 \log (d) d B$ \\
\hline $\begin{array}{l}\text { IMT2020 Path Loss (Urban NLOS } \\
\left.\text { at } f_{c}=700 \mathrm{MHz}\right)\end{array}$ & $190.434+39.09 \log (d) d B$ \\
\hline $\begin{array}{l}\text { IMT2000 Standard Deviation of } \\
\text { Log-normal Shadowing }\end{array}$ & $6 \mathrm{~dB}$ \\
\hline Path Loss 40 m from SCA & $127+30 \log (d) d B$ \\
\hline Power Amplifier Efficiency & $\frac{1}{\rho_{0}}=0.388, \frac{1}{\rho_{j}}=0.052 \forall j$ \\
\hline Circuit Power per Antenna & $\eta_{0}=12.85 \mathrm{~W}, \eta_{j}=0.8 \mathrm{~W} \forall j$ \\
\hline Constraint per Antenna & $q_{0, l}=66 \mathrm{~mW}, q_{j, l}=0.08 \mathrm{~mW}$ \\
\hline Number of Simulation/data point & 10 \\
\hline
\end{tabular}

\section{Performance Evaluation Metrics}

The performance evaluation of MM-SCA is done by calculating the total power consumption which can be defined as follows:

$$
P_{r}=\frac{\sum_{i=1}^{M} P_{r}^{i}}{M}
$$

where $P_{r}$ is the average received power at users, $P_{r}^{i}$ is the power received at user $i$ and $M$ denotes the total number of users.

\section{Simulation Results AND Discussions}

In this section, the simulation results of all scenarios are illustrated by mean values with $95 \%$ confident interval.

\section{(i) Urban Macro Cell of $3 G P P$}

Fig.2 and Fig.3 illustrate the average total power consumption per subcarrier $[\mathrm{dBm}]$ at BS and SCAs, respectively versus the number of antennas at BS. The QoS constraint considered here is $2 \mathrm{Bits} / \mathrm{sec} / \mathrm{Hz}$. According to Fig.2, it is obvious that the higher the number of antennas at SCA is, the lower the total power consumption per sub carrier becomes. When the number of BS antennas is large enough, the total power consumption per subcarrier is not relevant to the number of BS antennas. However, in case of Fig.3, it is apparent that the total power consumption per subcarrier of SCAs are almost the same for all number of BS antennas except the case of "No SCAs".

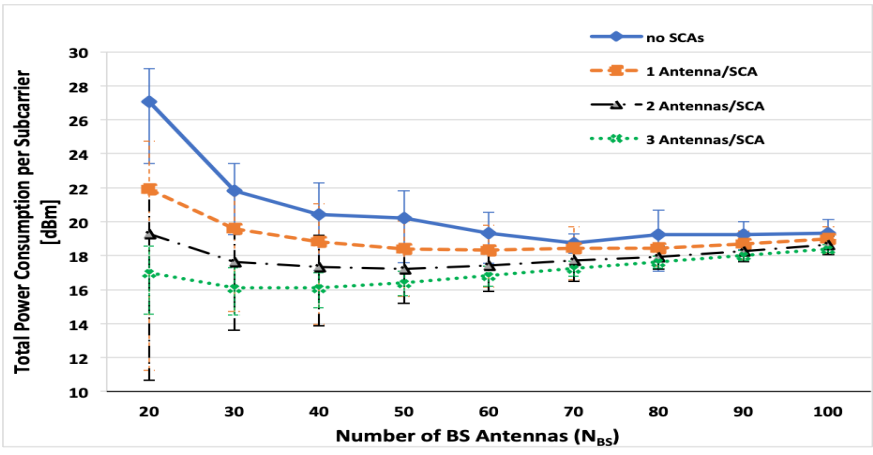

Fig. 2. Total Power Consumption per Subcarrier of BSs (Scenario-i)

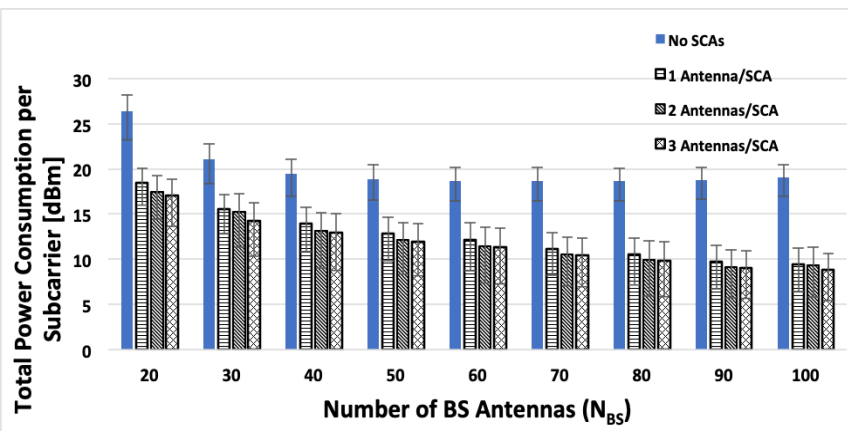

Fig. 3. Total Power Consumption per Subcarrier of SCAs (Scenario-i)

Fig.4 shows the average received power at users $[\mathrm{dBm}]$ with respect to QoS target per user $[\mathrm{Bits} / \mathrm{sec} / \mathrm{Hz}]$. It is apparent that the higher the information rate is, the higher the average received power becomes. However, the average received power becomes saturated when the information rate is high enough (higher than $2.5 \mathrm{bits} / \mathrm{sec} / \mathrm{Hz}$ ).

Fig.5 expresses the average total power consumption per subcarrier versus information rate. In this case, the $50 \mathrm{BS}$ antennas and 2 antennas/SCA are adopted. The three cases of performance are compared; only BS is present, low complexity algorithm [7] and our proposed MM-SCA system. It is obvious that our proposed MM-SCA has the lowest total power consumption among all methods.

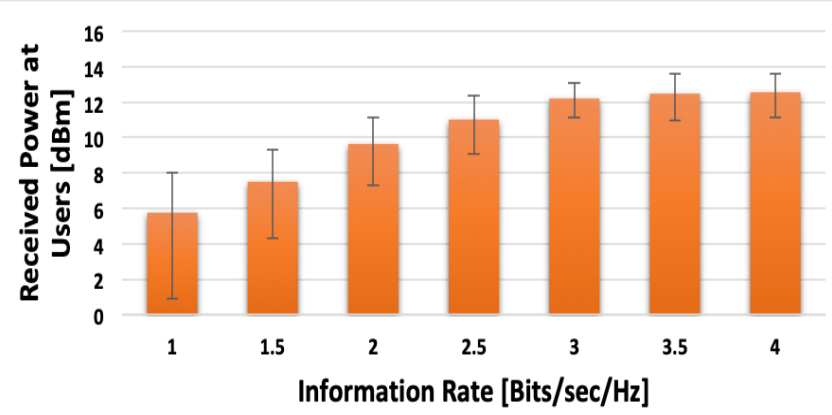

Fig. 4. Received Power at Users vs QoS Target (Scenario-i) 


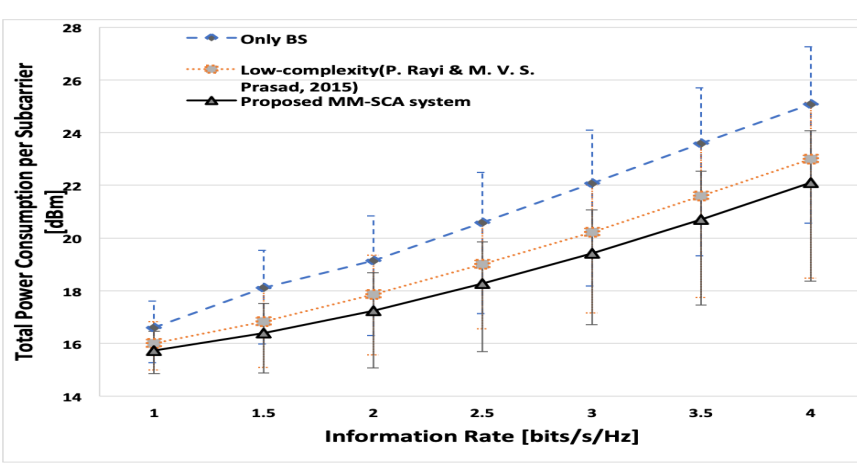

Fig. 5. Performance Comparison of Different Beam-forming Schemes (Scenario-i)

(ii) Urban Macro Cell Non-Line-of-Sight deployment under IMT 2020 standard with $f_{c}=2 \mathrm{GHz}$

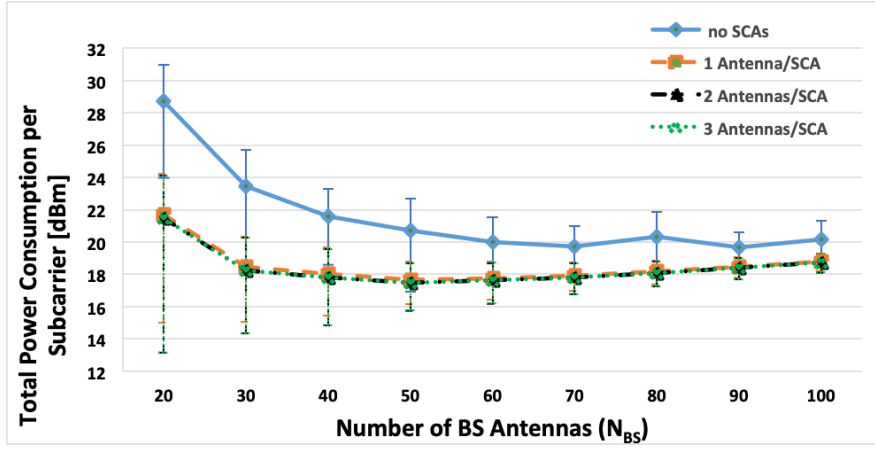

Fig. 6. Total Power Consumption per Sub-carrier of BSs (Scenario-ii)

Fig.6 depicts the same performance metric as Fig.2 but under IMT 2020 standard with a carrier frequency of $2 \mathrm{GHz}$. The results show the same trend as Fig.2. However, in this case, it is clear that the number of antenna per SCA has no impact on the total power consumption per carrier at all.

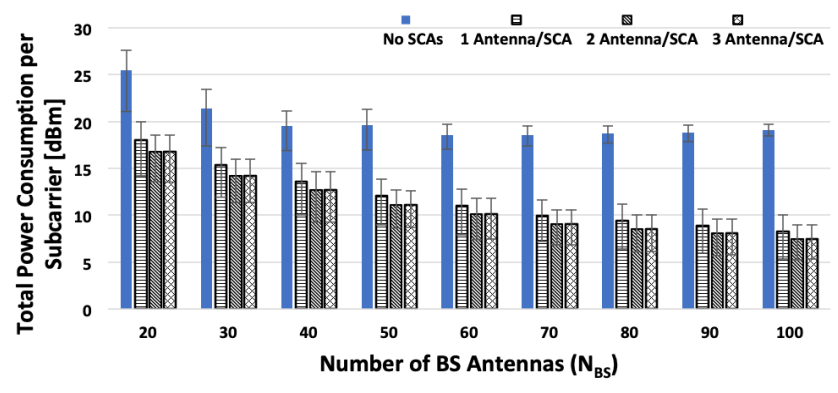

Fig. 7. Toal Power Consumption per Subcarrier of SCAs (Scenario-ii)

Similarly, Fig. $7 \sim$ Fig.9 depict the same performance metric as Fig.3 Fig.6, respectively but under IMT2020 standard with a carrier frequency of $2 \mathrm{GHz}$. It is obvious that the results are the same trend as in Fig.3 Fig.6, but with a little bit lower values.

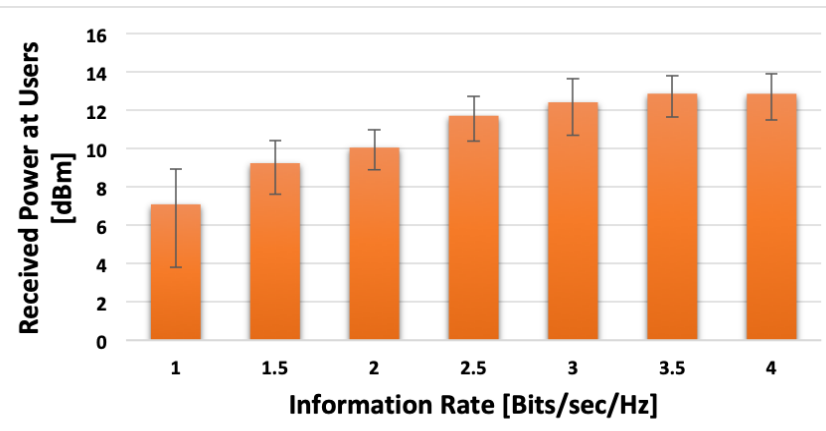

Fig. 8. Received Power at Users vs QoS Target (Scenario-ii)

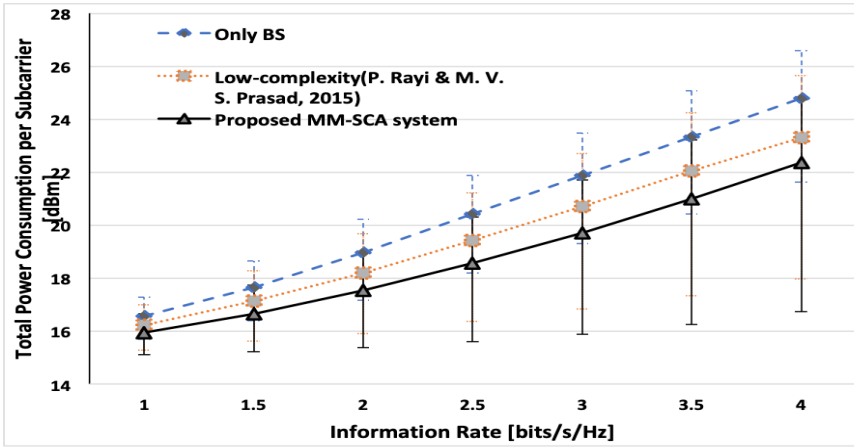

Fig. 9. Performance Comparison of Different Beam-forming Schemes (Scenario-ii)

(iii) Urban Macro cell Non-Line-of-Sight deployment under IMT 2020 standard with $f_{c}=700 \mathrm{MHz}$

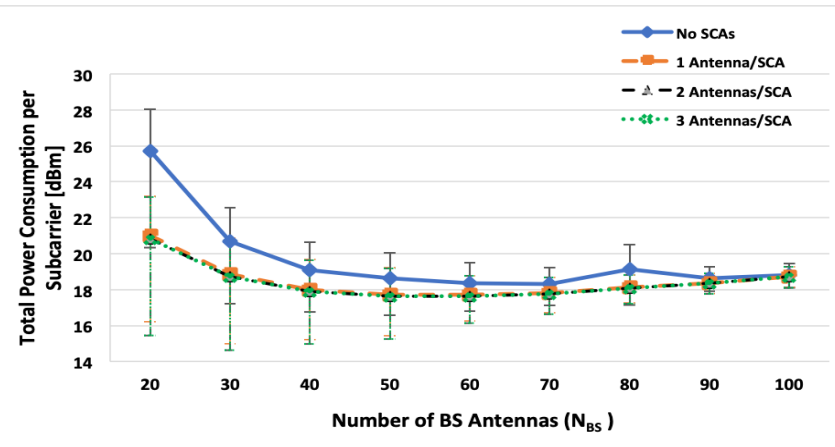

Fig. 10. Total Power Consumption per Subcarrier of BSs (Scenario-iii)

Fig.10 13 illustrate the same performance metrics as shown in Fig.6 9, but the carrier frequency is $700 \mathrm{MHz}$. All results show the same trend as shown in previously shown results, but with a little bit different values.

Finally, the performance comparison of our proposed method under 2 path loss model, namely 3GPP and IMT2020, is illustrated in Fig.14.

As shown in Fig.14, it is obvious that 3GPP path loss model provides a little bit lower power consumption per subcarrier at BS than IMT2020 at all information rate, regardless of the carrier frequency used in IMT2020. 


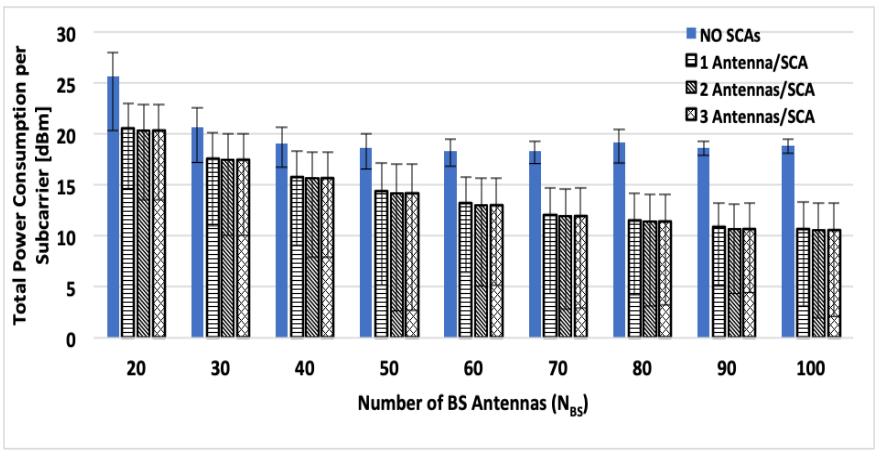

Fig. 11. Total Power Consumption per Subcarrier of SCAs (Scenario-iii)

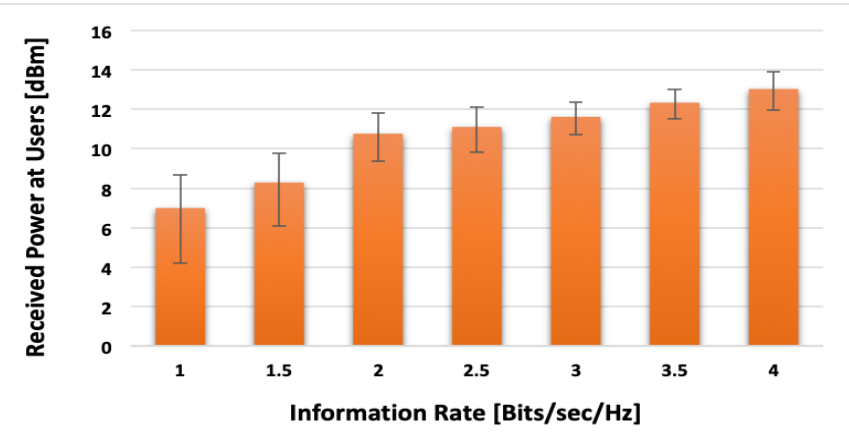

Fig. 12. Received Power at Users vs QoS Target (Scenario-iii)

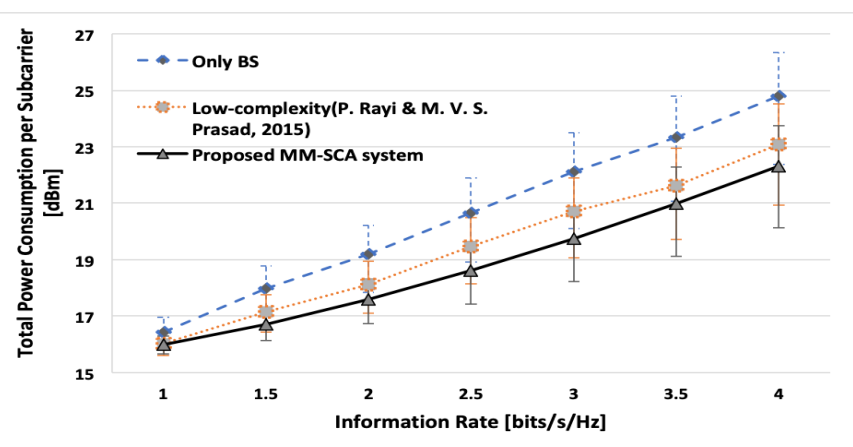

Fig. 13. Performance Comparison of Different Beam-forming Schemes (Scenario-iii)

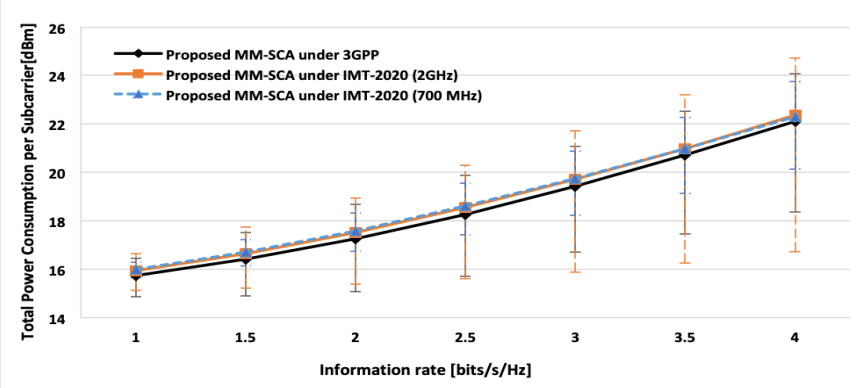

Fig. 14. Total Power Consumption per Subcarrier Comparison at BS of the Proposed Method under Two Path Loss Model

\section{CONClusions}

In this research, we investigate further the power optimization technique between the BS and SCA proposed in [2] under three scenarios; Urban Macro cell of 3GPP, Urban Macro cell NLOS under IMT2020 with carrier frequency of $2 \mathrm{GHz}$ and $700 \mathrm{MHz}$.

It can be concluded that in the case of $3 \mathrm{GPP}$ scenario optimal number of antennas at SCAs cannot be determined but it is clear that power consumption decreases when the number of antennas per SCA increases. We should keep in mind that if we gradually increase the number of antennas, the interference between SCA's antennas also increases. However, in case of IMT 2020 standards, it can be concluded that 1 antenna/SCA is optimal since to further increase of number of antenna does not bring any significant change in the power consumption. From all the scenarios it can be observed that the average received power at users increases when QoS target increases, whereas SCAs contribution decreases when the number of BS antennas increases. Apart from these the most important thing to be observed is significant difference of power consumption in the case of not using the SCAs and in the case of using the SCAs, which can be considered in building an energy efficient system. Finally, it is apparent that our proposed method has a little bit lower power consumption per subcarrier at BS at all information rate under 3 GPP path loss model than IMT2020 path loss model regardless of carrier frequency used.

\section{REFERENCES}

[1] Aditya Trivedi, Shweta Rajoria, and W. Wilfred Godfrey, "A comprehensive survey: Small cell meets massive MIMO", Physical communication, vol. 26, pp.40-49, February 2018. doi:https://doi.org/10.1016/j.phycom.2017.11.004.

[2] Emil Bjornson, Marios Kountouris and Luca Sanguinetti, "Deploying Dense Networks for Maximal Energy Efficiency: Small Cells Meet Massive MIMO", IEEE Journal on Selected Areas in Communications, 34(4), pp.832-847, April 2016.

[3] ERICSSON, "Ericcson energy and carbon report", ICT, 2014 https://www.ericsson.com/assets/local/about-ericsson/sustainabilityand-corporate-responsibility/documents/ericsson-energy-and-carbonreport.pdf

[4] International telecommunications union (ITU), (2017), Draft new report ITU-R M.[IMT-2020.EVAL].

[5] J. Sturn, "Using SeDuMi 1.02, a MATLAB toolbox for optimization over symmetric cones", Optimization Methods and Software, vol.11-12, pp.625-653, 1999.

[6] Emil Bjrnson, Marios Kountouris and Mrouane Debbah, "Massive MIMO and Small Cells: Improving Energy Efficiency by Optimal Soft-Cell Coordination", the IEEE International Conference on Telecommunications (ICT), pp.1-5, Morocco, May 2013. doi:10.1109/ICTEL.2013.6632074

[7] P. Rayi and M. V. S. Prasad, "Optimization of energy and spectral efficiency of massive MIMO-small cell system", the IEEE International Conference on Smart Technologies and Management for Computing, Communication, Controls, Energy and Materials (ICSTM), pp. 233-238, Chennai, 2015 doi:10.1109/ICSTM.2015.7225420

[8] Rakesh kumar Jha and Gupta, A.,"Power optimization using massive MIMO and small cells approach in different deployment scenarios", WIRELESS NETWORKS, 23(3), 959, 2017 doi:https://doi.org/10.1007/s11276-015-1174-y

[9] Vara Prasad Koppisetti and Naga Raghavendra Surya, "Massive MIMO for 5G wireless networks : an energy efficiency perspective", University of British Columbia, 2015 doi:10.14288/1.0300643

[10] X. Hou, X. Wang, H. Jiang and H. Kayama, "Investigation of massive MIMO in dense small cell deployment for 5G", the IEEE 84th Vehicular Technology, 2016 